\title{
Internal Mammary Artery Injury Related to Chest Compressions in a Patient with Post-cardiac Arrest Syndrome
}

\author{
Shoji Kawakami ${ }^{1,2}$, Teruo Noguchi ${ }^{1}$, Takahito Doi ${ }^{1}$, Yoshio Tahara ${ }^{1}$, Yoshihiro Sanda ${ }^{3}$, \\ Tetsuya Fukuda ${ }^{3}$, Hisao Ogawa ${ }^{1,4}$ and Satoshi Yasuda ${ }^{1,2}$
}

\begin{abstract}
Although high-quality cardiopulmonary resuscitation (CPR) is essential for survival from cardiac arrest, chest compressions can also sometimes lead to life-threatening chest injuries. In addition, post-cardiac arrest syndrome patients often have coagulopathy due to therapeutic hypothermia, mechanical hemodynamic support, or both. Therefore, when progressive anemia and prolonged shock are detected in patients who have received CPR, identifying the cause of hemorrhagic shock is crucial. We herein present an interesting case of hemorrhagic shock due to an internal mammary artery injury secondary to CPR that was detected by computed tomography and invasive angiography.
\end{abstract}

Key words: internal mammary artery injury, cardiopulmonary resuscitation, post-cardiac arrest syndrome

(Intern Med 55: 1299-1303, 2016)

(DOI: 10.2169/internalmedicine.55.5762)

\section{Introduction}

The 2010 American Heart Association Guidelines for Cardiopulmonary Resuscitation and Emergency Cardiovascular Care emphasize high-quality cardiopulmonary resuscitation (CPR) as the cornerstone for survival from cardiac arrest. While the 2005 guidelines recommended compressions to a depth of 38 to $50 \mathrm{~mm}$, the 2010 guidelines recommend a depth of $\geq 50 \mathrm{~mm}$ with no upper limit specified $(1,2)$. However, there is an increased risk of complications with the deeper compressions recommended by this new guideline (3). Several studies have shown that computed tomography (CT) can be useful for detecting various chest injuries arising as complications associated with CPR (4-6). In the emergency department, identifying the presence of active bleeding is one of most important steps in the management of acute trauma. Contrast enhanced CT can detect sites of active bleeding when a patient is in hemorrhagic shock. It is also useful for deciding between surgical or radiological in- tervention for achieving hemostasis.

We herein present a case of hemorrhagic shock caused by an internal mammary artery (IMA) injury related to CPR that was detected by $\mathrm{CT}$ and invasive angiography.

\section{Case Report}

A 77-year-old man with a mechanical mitral valve and left ventricular dysfunction (left ventricular ejection fraction, $39 \%$ ) was transferred to our emergency department due to sustained ventricular fibrillation (VF) for at least 33 minutes despite undergoing continuous CPR that included manual chest compressions and electrical defibrillation by emergency medical service personnel.

On admission, since the intravenous administration of amiodarone was ineffective in terminating $\mathrm{VF}$, arteriovenous bypass via venoarterial extracorporeal membrane oxygenation (VA-ECMO) was quickly introduced (door to ECMO time, 23 minutes). The VA-ECMO flow was established with right femoral venous cannulae for withdrawing deoxy-

\footnotetext{
${ }^{1}$ Department of Cardiovascular Medicine, National Cerebral and Cardiovascular Center, Japan, ${ }^{2}$ Department of Advanced Cardiovascular Medicine, Graduate School of Medical Sciences, Kumamoto University, Japan, ${ }^{3}$ Department of Radiology, National Cerebral and Cardiovascular Center, Japan and ${ }^{4}$ Department of Cardiovascular Medicine, Graduate School of Medical Sciences, Kumamoto University, Japan

Received for publication May 13, 2015; Accepted for publication August 4, 2015

Correspondence to Dr. Teruo Noguchi, tnoguchi@hsp.ncvc.go.jp
} 

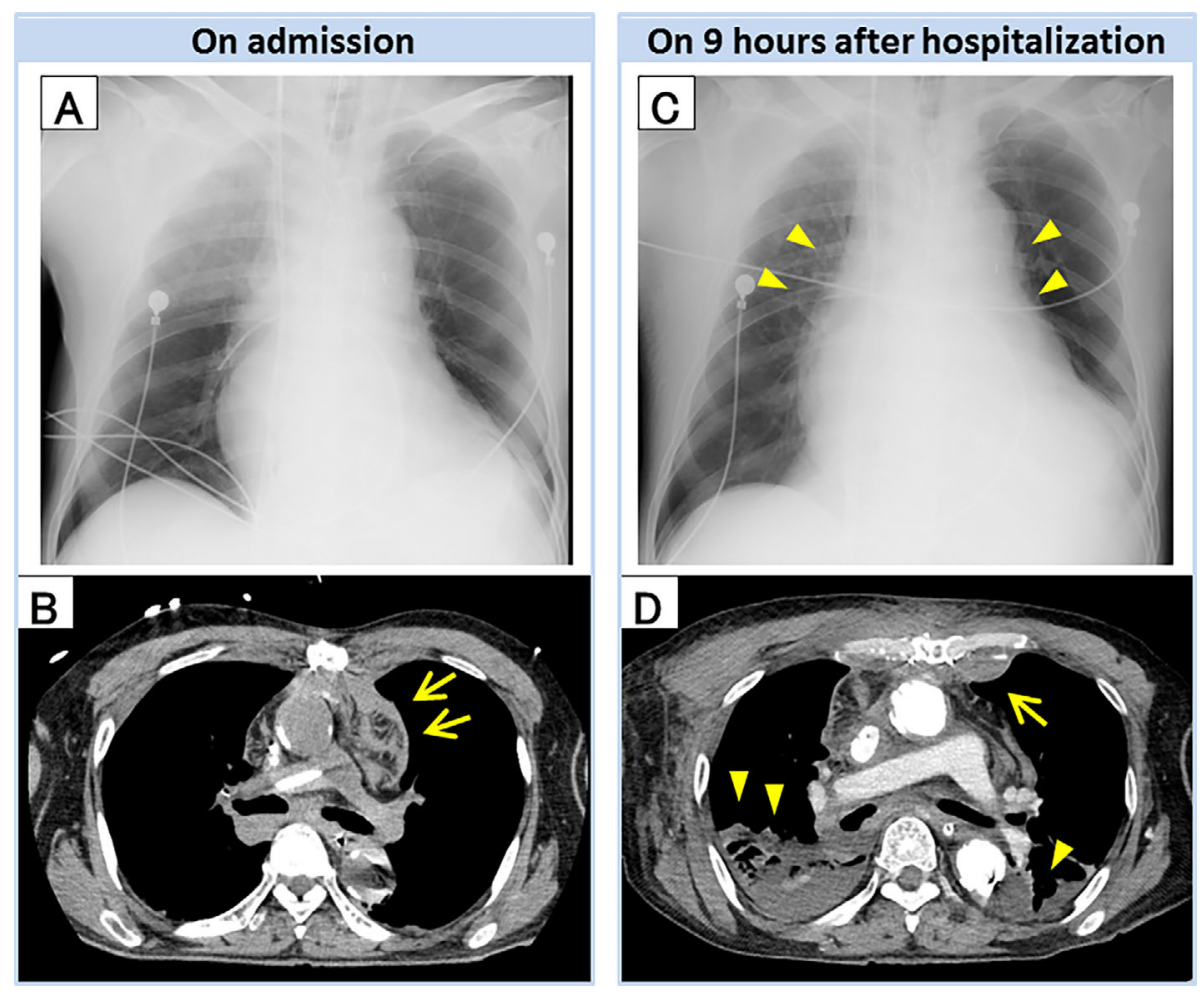

Figure 1. Chest radiography and computed tomography on and 9 hours after hospital admission. On admission, immediately after the introduction of mechanical circulatory support, (A) chest radiography showed a mild widening of the mediastinal silhouette and (B) non-contrast computed tomography (CT) revealed retrosternal hemorrhaging localized to the mediastinal space between the sternum and the heart (arrows). Nine hours after admission, the mediastinal silhouette was larger on chest X-ray (C, arrowheads). Contrast enhanced CT revealed more retrosternal hemorrhaging with extravasated contrast material (D, arrow) and hemothorax (D, arrowheads). $A$ and $C$ were taken in the supine position.

genated venous blood from the right atrium and left femoral arterial cannulae for returning oxygenated arterial blood. VF was terminated after additional electrical defibrillation following the initiation of VA-ECMO. Subsequent urgent coronary angiography revealed intermediate stenosis in the left main trunk and severe stenosis at the ostium of the left circumflex artery. Percutaneous coronary intervention was not performed due to the absence of a limited coronary artery flow and a high risk of bleeding due to the long duration of chest compressions and the administration of dual antiplatelet therapy. Intraaortic balloon pumping (IABP) was established via cannulae inserted into the right femoral artery. Cinefluoroscopy showed no significant insufficiency of the prosthetic mitral valve. Chest radiography showed a slight widening of the mediastinal silhouette (Fig. 1A). Noncontrast CT revealed retrosternal hemorrhaging localized to the mediastinal space between the sternum and the heart due to fractures in the sternum and multiple ribs (Fig. 1B). At this point, enhanced CT to detect any active bleeding was not performed because the patient was hemodynamically unstable on circulatory support consisting of VA-ECMO, therapeutic hypothermia, and IABP. However, therapeutic hypothermia was discontinued after 9 hours due to hypotension. Despite the transfusion of red blood cells, platelets, and frozen fresh plasma, his vital signs remained unstable and coagulopathy persisted. Chest radiography showed a larger mediastinal silhouette (Fig. 1C). Therefore, we performed contrast enhanced CT. Contrast material was administered as a bolus of $79 \mathrm{~mL}$ at $2.6 \mathrm{~mL}$ per second via a right jugular venous catheter. CT at 70 seconds after contrast administration revealed additional retrosternal hemorrhaging with contrast material extravasation and hemothorax (Fig. 1D, 2); therefore, we suspected an injury to the left IMA. Selective transcatheter angiography demonstrated extravasation from a branch of the left IMA (Fig. 3). Selective transcatheter arterial embolization with Spongel ${ }^{\circledR}$ cut-off gelatin sponges (Astellas Pharma, Tokyo, Japan) was performed. After achieving hemostasis, the patient's anemia improved immediately and his hemodynamic parameters stabilized. ECMO and IABP were successfully weaned on hospital days 3 and 4, respectively. The patient was discharged from our hospital and transferred to a rehabilitation clinic on day 61. His cerebral performance category upon discharge from our hospital was 3 .

\section{Discussion}

IMA injury secondary to blunt chest trauma is a rare en- 

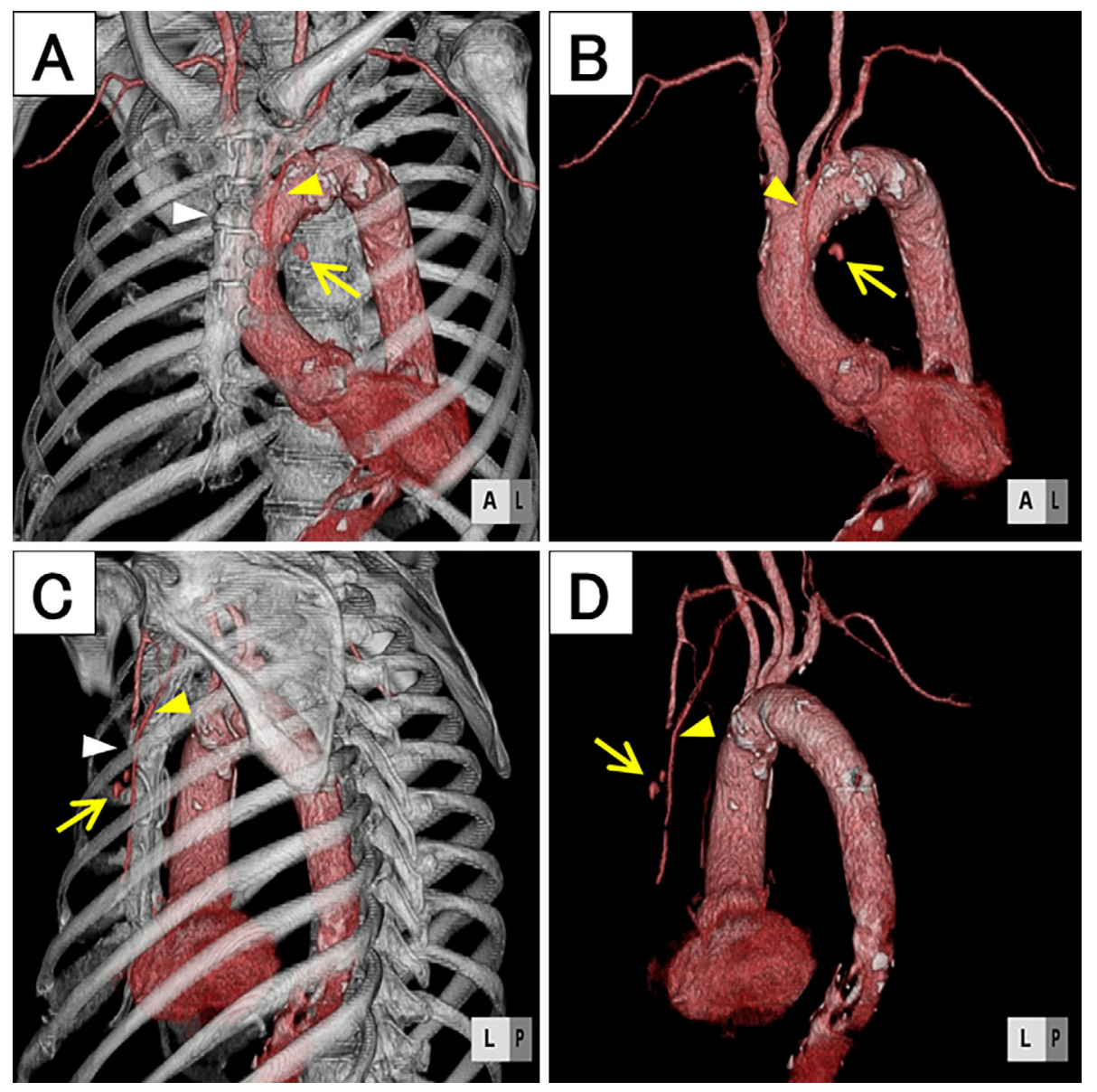

Figure 2. Bleeding from a left internal mammary artery injury on reconstructed 3-dimensional computed tomography images. Three-dimensional contrast enhanced CT showed contrast media extravasation (yellow arrows) close to the left internal mammary artery (yellow arrowheads). The location of the sternal fracture is indicated by a white arrowhead. Left anterior oblique views are shown in (A) and (B). (C) and (D) are left posterior oblique views. (A) and (C) are merged with bone images. CT indicates computed tomography.

tity but it is often life-threatening since it can progress to mediastinal hematoma with cardiac compression or hemothorax with hemorrhagic shock $(7,8)$. Although the presence of an anterior mediastinal hematoma implies IMA injury, a mediastinal hematoma secondary to blunt chest trauma can be due to injuries of other vessels including the aortic arch, intercostal, internal thoracic, and small mediastinal vessels and rib or sternum fractures (7). Thoracotomy and endovascular intervention are therapeutic options for IMA injury. In a review article of 49 patients with IMA injury due to blunt chest trauma, 22 patients (45\%) underwent surgery and 20 patients $(41 \%)$ underwent transcatheter embolization (9). The possible mechanisms of IMA injury include a fracture of an adjacent bony structure, such as a rib, clavicle, or sternum or shearing forces acting on the vessel during extreme acceleration or deceleration (10). Although there are no clinical studies of IMA injury related to CPR, the incidence of mediastinal hematoma due to chest compressions with a depth of 4 to $5 \mathrm{~cm}$ is reported to be $10 \%$ (11). In this particular patient with postoperative adhesions between intrathoracic blood vessels, sustained chest compressions may have led to IMA injury. In addition, coagulopathy secondary to post-cardiac arrest syndrome (PCAS), therapeutic hypothermia, anticoagulation for the mechanical heart valve, and mechanical circulatory support are precipitating factors for hemorrhage.

In this case, contrast enhanced CT was useful for detecting CPR-related IMA injury. CT is rapidly becoming the standard modality for assessing cardiovascular disorders such as aortic dissection (12) and pulmonary embolus (13) due to its greater spatial and temporal resolution. In brief CT angiography is usually recommended for assessing active arterial extravasation in the presence of vascular injury. Since the presence of a mediastinal hematoma and its anatomical location suggested a vascular origin, CT can be useful for distinguishing between angiographic and surgical intervention $(9,14,15)$. However, in patients with specific hemodynamic changes due to severely impaired left ventricular function and mechanical circulatory support, it can be difficult to optimize contrast enhancement and diagnostic imaging quality. In patients on VA-ECMO, injecting contrast into a blood vessel in an extremity is not recommended due 


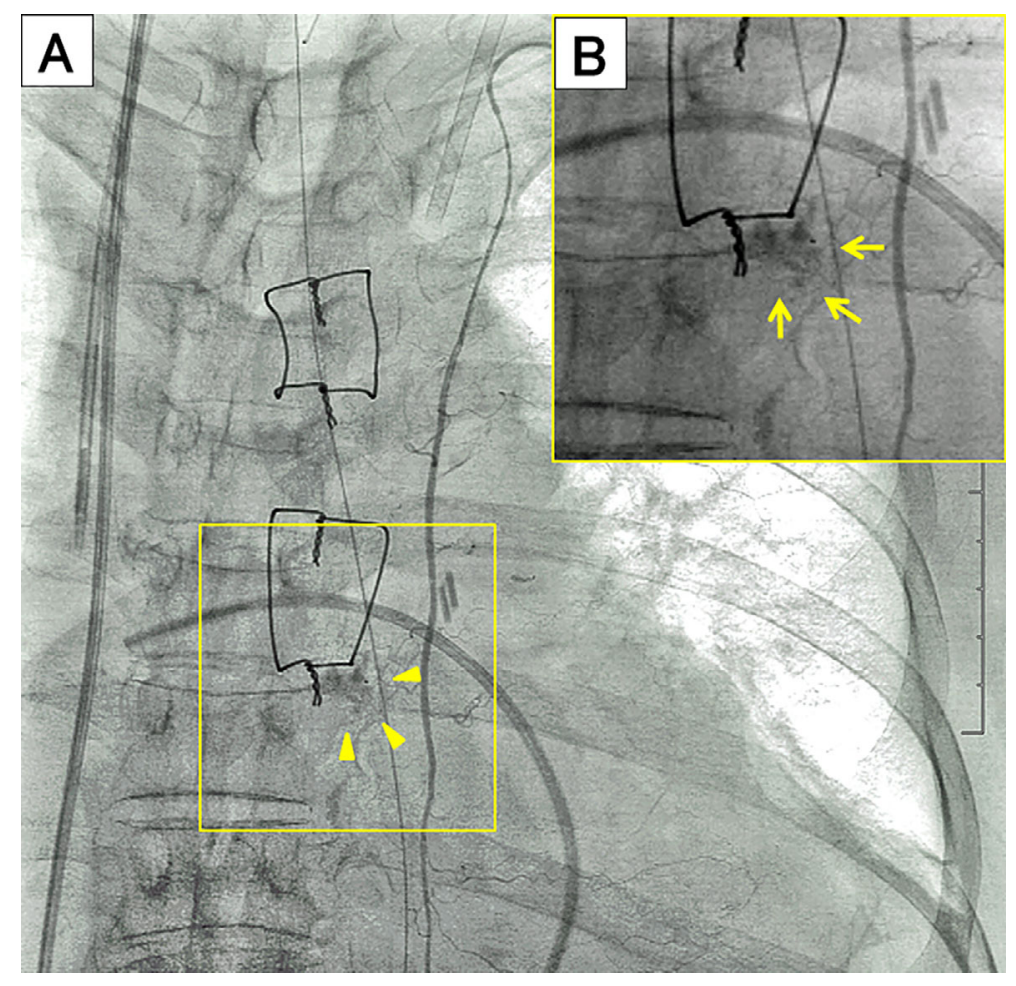

Figure 3. Selective transcatheter angiography. Selective transcatheter angiography showed extravasation from the branches of the left internal mammary artery at low (A, arrowheads) and high magnifications (B, arrows).

to insufficient circulation (16). When performing CT angiography to detect vascular injuries in patients with PCAS on mechanical circulatory support, contrast medium should be administrated via a central vein in the upper body and several scans should then be performed to determine the suitable contrast conditions for obtaining high-resolution images. Indeed, in this case, we re-scanned at 70 seconds after contrast injection to assess extravasation behind the sternum because images at 30 seconds after bolus injection through the right jugular vein were inadequate for detecting extravasation.

Although high-quality CPR is essential for survival from cardiac arrest, chest compressions can lead to lifethreatening chest injuries. Since patients with PCAS have a high risk of bleeding, especially those undergoing mechanical circulatory support or therapeutic hypothermia, the occurrence of retrosternal hemorrhaging due to IMA injury should be considered in PCAS patients with anemia refractory to transfusion.

The authors state that they have no Conflict of Interest (COI).

\section{References}

1. Travers AH, Rea TD, Bobrow BJ, et al. Part 4: CPR overview: 2010 American Heart Association Guidelines for Cardiopulmonary Resuscitation and Emergency Cardiovascular Care. Circulation 122: S676-S684, 2010.

2. Sayre MR, Koster RW, Botha M, et al. Part 5: Adult basic life support: 2010 International Consensus on Cardiopulmonary Resuscitation and Emergency Cardiovascular Care Science With Treatment Recommendations. Circulation 122: S298-S324, 2010.

3. Hellevuo H, Sainio M, Nevalainen R, et al. Deeper chest compression: more complications for cardiac arrest patients? Resuscitation 84: 760-765, 2013.

4. Kim EY, Yang HJ, Sung YM, et al. Multidetector CT findings of skeletal chest injuries secondary to cardiopulmonary resuscitation. Resuscitation 82: 1285-1288, 2011.

5. Kim MJ, Park YS, Kim SW, et al. Chest injury following cardiopulmonary resuscitation: a prospective computed tomography evaluation. Resuscitation 84: 361-364, 2013.

6. Hoke RS, Chamberlain D. Skeletal chest injuries secondary to cardiopulmonary resuscitation. Resuscitation 63: 327-338, 2004.

7. Braatz T, Mirvis SE, Killeen K, Lightman NI. CT diagnosis of internal mammary artery injury caused by blunt trauma. Clin Radiol 56: 120-123, 2001.

8. Madoff DC, Brathwaite CE, Manzione JV, et al. Coexistent rupture of the proximal right subclavian and internal mammary arteries after blunt chest trauma. J Trauma 48: 521-524, 2000.

9. Chen JM, Lv J, Ma K, Yan J. Assessment of internal mammary artery injury after blunt chest trauma: a literature review. J Zhejiang Univ Sci B 10: 864-869, 2014.

10. Husted JW, Stock JR, Manella WJ. Traumatic anterior mediastinal hemorrhage: control by internal mammary artery embolization. Cardiovasc Intervent Radiol 5: 268-270, 1982.

11. Smekal D, Lindgren E, Sandler H, Johansson J, Rubertsson S. CPR-related injuries after manual or mechanical chest compressions with the LUCAS ${ }^{\mathrm{TM}}$ device: a multicenter study of victims after unsuccessful resuscitation. Resuscitation 12: 1708-1712, 2014.

12. JCS Joint Working Group. Guidelines for diagnosis and treatment of aortic aneurysm and aortic dissection (JCS 2011): digest version. Circ J 77: 789-828, 2013.

13. Sakuma M, Nakamura M, Nakanishi N, et al. Clinical characteris- 
Intern Med 55: 1299-1303, 2016 DOI: 10.2169/internalmedicine.55.5762

tics, diagnosis and management of patients with pulmonary thromboembolism who are not diagnosed in the acute phase and not classified as chronic thromboembolic pulmonary hypertension. Circ J 69: 1009-1015, 2005.

14. Richardson P, Mirvis SE, Scorpio R, Dunham CM. Value of CT in determining the need for angiography when findings of mediastinal hemorrhage on chest radiographs are equivocal. AJR Am J Roentgenol 156: 273-279, 1991.
15. Mirvis SE, Shanmuganathan K, Buell J, Rodriguez A. Use of spiral computed tomography for the assessment of blunt trauma patients with potential aortic injury. J Trauma 45: 922-930, 1998.

16. Liu KL, Wang YF, Chang YC, et al. Multislice CT scans in patients on extracorporeal membrane oxygenation: emphasis on hemodynamic changes and imaging pitfalls. Korean J Radiol 15: 322-329, 2014.

(C) 2016 The Japanese Society of Internal Medicine http://www.naika.or.jp/imonline/index.html 